\title{
Layered Structures of Ti-6Al-4V Alloy and Metal Matrix Composites on Its Base Joint by Diffusion Bonding and Friction Welding
}

SV Prikhodko ${ }^{1 *}$, DG Savvakin ${ }^{2}$, PE Markovsky ${ }^{2}$, OO Stasiuk ${ }^{2}, \mathrm{~N}_{\text {Enzinger }}^{3}, \mathrm{~F} \mathrm{Deley}^{4}$, B Flipo $^{5}$, AA Shirzadi ${ }^{6,7}$, HM Davies ${ }^{8}$, PD Davies ${ }^{8}$, J Penny ${ }^{1}, \mathrm{~K}_{\text {Bozhilov }}^{9}$ and OM Ivasishin ${ }^{2}$

1. Department of Materials Science and Engineering, University of California Los Angeles, Los Angeles, CA, USA.

2. G.V. Kurdyumov Institute for Metal Physics, National Academy of Science of Ukraine, Kiev, Ukraine.

3. Institute of Materials Science, Joining and Forming, Graz University of Technology, Graz, Austria.

4. Taylor-Winfield Technologies, Youngstown, OH, USA.

5. TWI, Granta Park, Great Abington, Cambridge, UK.

6. School of Engineering and Innovation, The Open University, Milton Keynes, UK.

7. Department of Materials Science and Metallurgy, University of Cambridge, Cambridge, UK.

${ }^{8 .}$ Institute of Structural Materials' Swansea University Bay Campus, Crymlyn Burrows, Fabian Way, Swansea, UK.

9. Central Facility for Advanced Microscopy and Microanalysis, University of California Riverside, Riverside, CA, USA.

* Corresponding author: sergey@seas.ucla.edu

Metallic layered structures demonstrate an advanced set of characteristics that combine different properties not found within homogenous bulk materials. Powder metallurgy (PM) is proven to be the most efficient way of fabrication of layered structures, including highly rated structures of Ti alloys. Residual porosity, however, remains one of the biggest problems of titanium-based PM products and this can adversely affect the mechanical properties and performance of the structural parts. Post-sintering hot deformation is a common way to control the porosity of metallic materials. Traditional thermomechanical processing like hot rolling, however, could not be applied on multi-layered structures due to the disparity of the different layers' plastic flow [1]. Separate processing of high performance individual layers to reach their best parameters, followed by post processing bonding of the mating subcomponents is a credible pathway for fabrication of the layered materials with highly optimized properties of each individual layer. In this study we used diffusion bonding (DB) and friction welding to join the parts made of Ti-6Al-4V alloy and metal matrix composites on the base of this alloy reinforced with $10 \%$ of either TiB or $\mathrm{TiC}$. Parts were fabricated using blended elemental PM. Different protocols were used to join the materials: DB welding via rotational friction (RFW) and linear friction (LFW) as well as different geometries of mating subcomponents were tested. Structure characterization of the joints using light optical microscopy, SEM, EDS, EBSD as well as mechanical tests were performed. All used protocols were generally successful in bonding the parts made of Ti-64 alloy and composites on its base. The potential of DB, RFW and LFW of Ti-6Al-4V alloy and its MMC are discussed [2].

References:

[1] SV Prikhodko et al., Materials Science Forum 941 (2018), p. 1384.

doi:10.4028/www.scientific.net/MSF.941.1384

[2] The following authors SVP, DGS, PEM, OOS, JP, OMI acknowledge funding from the NATO Agency Science for Peace and Security (\#G5030). 

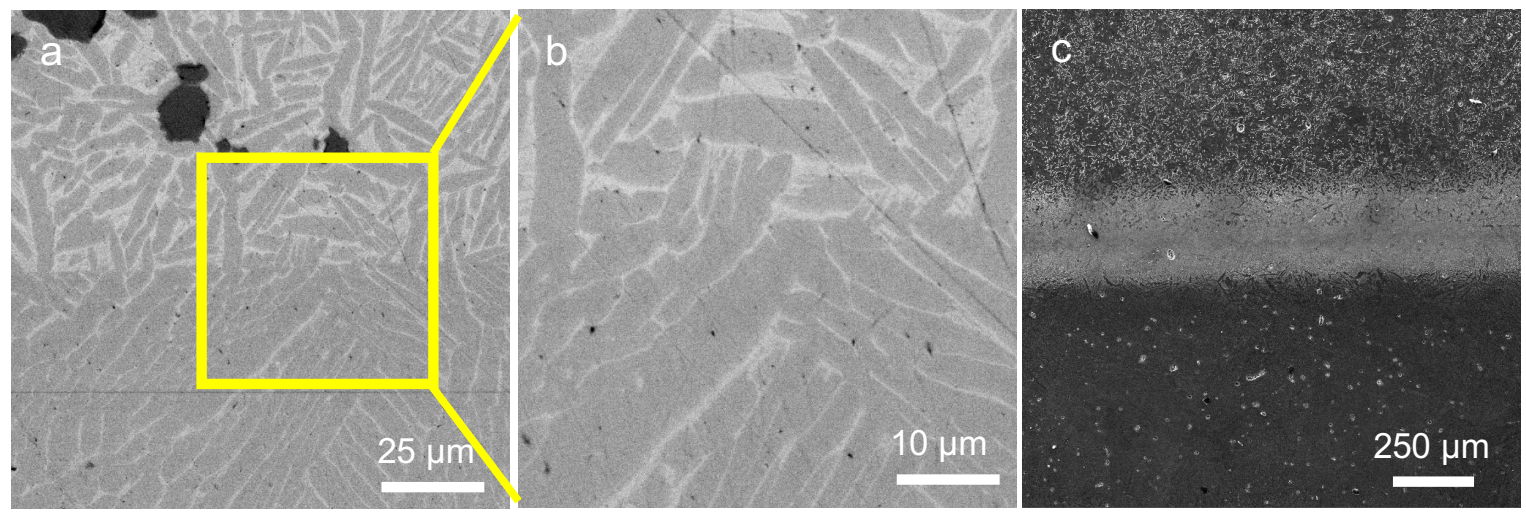

Figure 1. SEM images of the interface structure between the alloy (bottom) and composite (top) reinforced with $\mathrm{TiC}(\mathrm{a}, \mathrm{b})$ and $\mathrm{TiB}(\mathrm{c})$ after diffusion bonding for $1 \mathrm{~h}$ : at $850-900{ }^{\circ} \mathrm{C}, 1 \mathrm{MPa}(\mathrm{a}, \mathrm{b})$ and $1000^{\circ} \mathrm{C}, 0.7 \mathrm{MPa}(\mathrm{c})$. Images show complete integration between the layers. Incept on (b) depicts some $\alpha$-Ti crystallites infiltrate through the interface. Depending on the processing conditions the thickness of the joint interface varied from sub- to hundreds of microns.
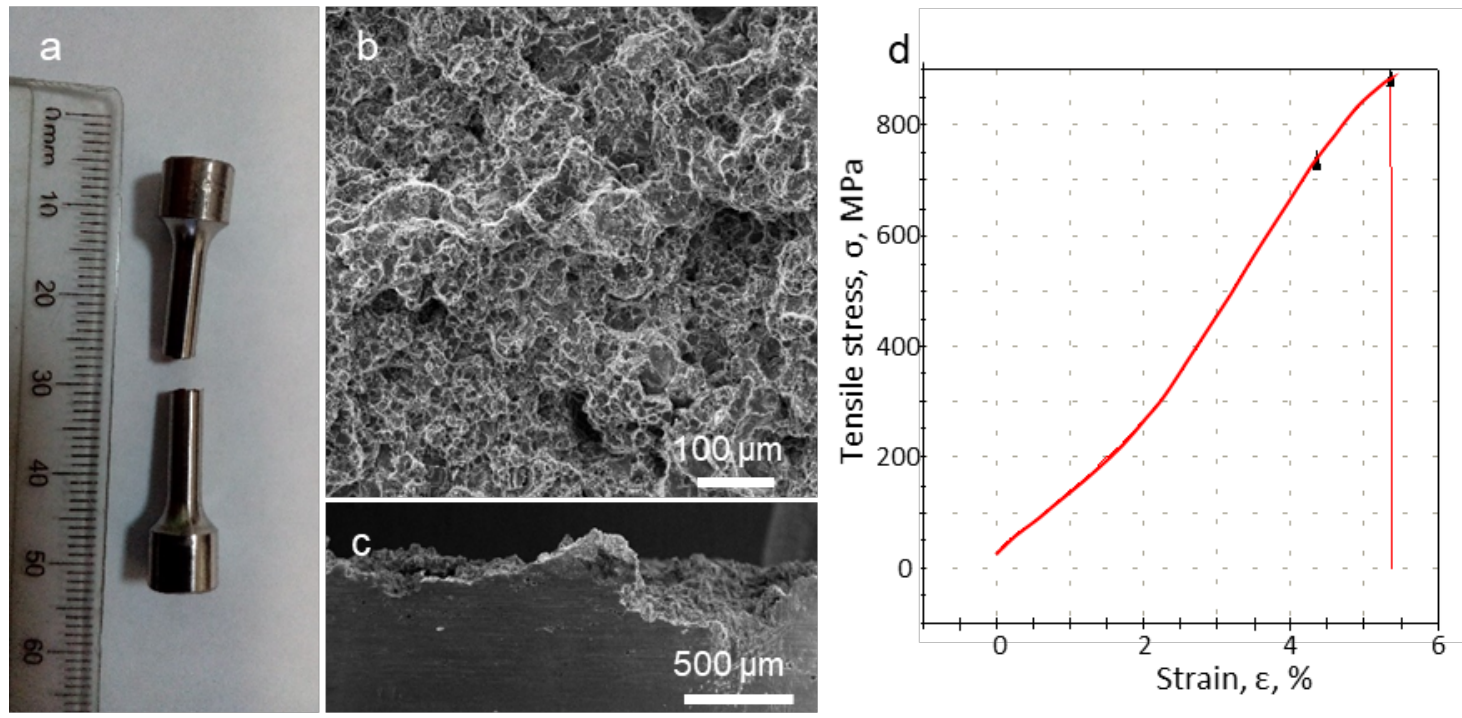

Figure 2. Tensile test experiment (ASTM E8-M) of the sample after bonding using LFW. Bonding pair: Ti-64 alloy and Ti-64+10\% TiC composite. Welding parameters: area $50 \mathrm{~mm}^{2}$; friction amplitude, $3.63 \mathrm{~mm}$; interface pressure, $110 \mathrm{MPa}$. Sample after the test (a), fracture (b), side view of the fractured area (c) and tensile test chart (d). Fracture occurs outside of the weld area within the composite layer. The UTS is close to $900 \mathrm{MPa}$, elongation 5.36\%. Measured UTS is close to this value of the sintered Ti64 alloy (960-1030MPa) and significantly higher than Ti-64+10\% TiC composite (620 $\mathrm{MPa})$. 